\title{
NUMERICAL SIMULATION OF COMPRESSIBLE SQUEEZED-FILM DAMPING
}

\author{
Yao-Joe Yang and Stephen D. Senturia \\ Microsystems Technology Laboratory, Massachusetts Institute of Technology \\ Cambridge, MA 02139
}

\begin{abstract}
This paper presents the simulation of isothermal compressible squeezed-film damping effects for MEMS devices. The squeezedfilm goveming equation, the isothermal Reynold's equation, is widely used in lubrication theory. Both damping and compressibility effects of the squeezed film are modeled numerically. Small and large amplitude motions are simulated by using finite-element and finite-difference codes respectively. Macro-model examples with this squeeze-film effect are also demonstrated. We find that the air compressibility effect is significant in high frequency operation for small amplitude oscillations, and that the nonlinearity of the governing equation causes a nonlinear pressure response for large amplitude motion.
\end{abstract}

\section{INTRODUCTION}

MEMS devices are often operated in ambient pressure, so that air functions as an important working fluid. An air film between two closely spaced plates moving in normal relative motion gives rise to a pressure distribution over the plate. The total pressure force, which opposes the motion of the plate, is known as squeezefilm damping. Fig. 1 shows the schematic view of squeeze-film damping. Theory $[1,2]$ shows that the viscous damping effect of the air film dominates at low frequencies, but spring-like behavior takes over at higher frequencies. The experimental studies [3-7] are in general agreement with the theory, but most of these studies focused only on the behavior of small amplitude oscillations. In this paper, we demonstrate that these effects can be successfully modeled numerically under small-amplitude sinusoidal conditions by a finite element package, as well as under large amplitude motion by finite difference codes. A simulated macromodel including the squeeze-film damping effect for a microaccelerometer [8] with small amplitude motions is presented. The frequency response shows that the spring effect of the squeeze film due to air compression shifts the resonance. Simulations of large amplitude sinusoidal motions show that the nonlinear effects of the pressure force are dramatic.

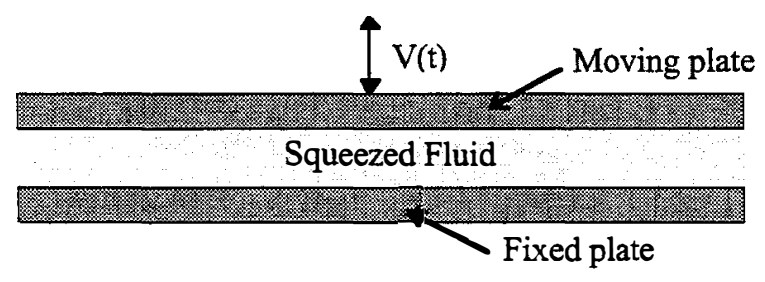

Fig. 1 Typical squeeze film geometry.

\section{ISOTHERMAL REYNOLD'S EQUATION}

Parallel rigid plates in relative normal motion experience a back-force from the time-dependent pressure distribution in the gas film. The product of the plate velocity and this back-force is the power transferred into the gas film by the plate motion, some of which appears as damping, the rest as stored energy in the compressed gas. The governing equation, the compressible isothermal Reynold's equation, is a time-dependent non-linear parabolic partial differential equation. The non-dimensional form of this equation for normal relative motion can be written as

$$
\nabla \cdot\left(P H^{3} \nabla P\right)=\sigma^{*} \frac{\partial(P H)}{\partial t}
$$

where

$$
H=\frac{h}{h_{0}} ; P=\frac{p}{P_{a}} ; \quad \sigma^{*}=\frac{12 \mu L^{2}}{P_{a} h_{0}^{2}}
$$

$h$ is gap thickness, $h_{0}$ is the initial gap thickness, $p$ is pressure, $P_{a}$ is ambient pressure, $L$ is plate length, $\mu$ is viscosity, and $\sigma^{\circ}$ is a characteristic time [1].

The Reynold's equation is obtained from the Navier-Stokes equation, the continuity equation and the equation of state by assuming that (1) the inertia effect in the Navier-Stokes equation is negligible, (2) the pressure distribution across the gap is uniform and (3) the fluid velocity component perpendicular to plate surface is negligible.

\section{LINEARIZED MODEL}

Since the isothermal Reynold's equation is a highly non-linear partial differential equation, in general it has to be solved numerically. However, this equation can be linearized into a diffusion-like partial differential equation assuming (1) small gap thickness, (2) low Reynold's number, (3) isothermal conditions, (4) small motion amplitude, and (5) small pressure variation. The non-dimensional form of the linearized Reynold's Equation is

$$
\begin{aligned}
& \nabla^{2} \Theta-\sigma \frac{\partial \Theta}{\partial \tau}=\sigma \frac{\partial e}{\partial \tau} \\
& \text { where } \\
& \tau=\omega t ; e=\frac{\delta}{h} \cos \omega t \\
& \Theta=\frac{\Delta p}{P_{a}} ; \sigma=\frac{12 \mu L^{2} \omega}{P_{a} h_{0}^{2}}
\end{aligned}
$$


$\omega$ is oscillation frequency, $\tau$ is non-dimensional time, $\delta$ is amplitude of oscillation, $\Delta p$ is small variation of pressure, $\Theta$ is the linearized non-dimensional pressure, and $\sigma$ is the squeeze number [1].

The key parameter in the linearized Reynold's Equation is the squeeze number $\sigma$, which measures the compression of the fluid in the gap. If $\sigma$ is close to 0 (low speed or frequency); the air film obeys nearly incompressible viscous flow; if $\sigma$ goes to infinity (at very high speed or oscillation frequency), the fluid is essentially trapped in the gap and behaves like a spring. The linearized analytical solution for sinusoidal motion is available for rectangular and circular plates [2]. Furthermore, since the linearized Reynold's equation is in the same form of the transient energy-flow equation, we can use the finite element fluidsmodeling package FIDAP [9] to generate the film pressure distribution for complex plate geometries by mapping the temperature variable in the energy equation to the pressure variable in the linearized Reynold's equation. Fig 2 shows analytical and numerical results of the non-dimensional pressure forces vs. squeeze number for an air film between two square plates with one plate in small-amplitude sinusoidal motion. The sinusoidal backforce on the plate has two components: one is in phase with plate velocity (the viscous damping force), the other is in phase with plate displacement (the spring force due to the compressibility of air). At small squeeze number (low frequency), the viscous force dominates because the air can squeeze out of the gap without being compressed. At large squeeze number (high frequency), the spring force increases because of compression of the air film. The numerical results for the spring force component agree with the analytical results, but the damping force component is about $8 \%$ lower than the analytical results at higher squeeze number. The reason for this small discrepancy is not yet understood, which places a limit of confidence of about $10 \%$ on the numerical results which follow. The normalized transient response for the total back-force is shown in Fig. 3. The forces have been normalized to their steady state maximum values. The force lags behind the sinusoidal plate velocity, and the phase lag increases with squeeze number because of the air compressibility effect. Seidel observed this lag experimentally, but chose to model it with a fixed delay instead of as a compression effect [4].

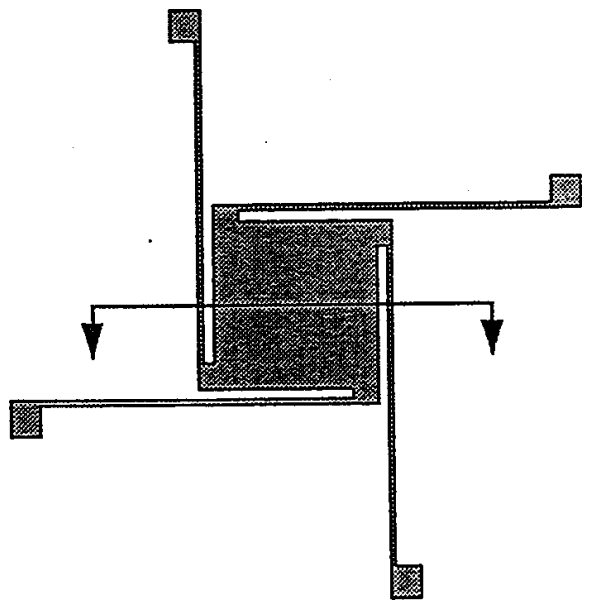

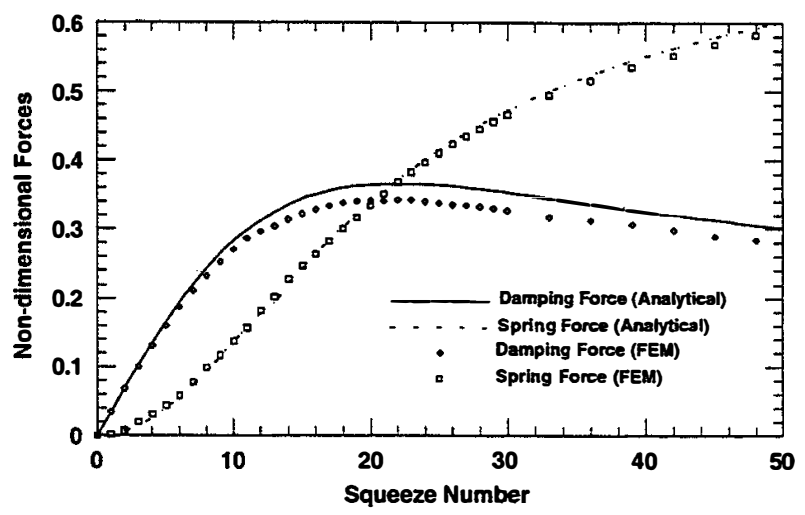

Fig. 2 Analytical and numerical results of non-dimensional damping and spring forces vs. squeeze number for $a$ square plate.

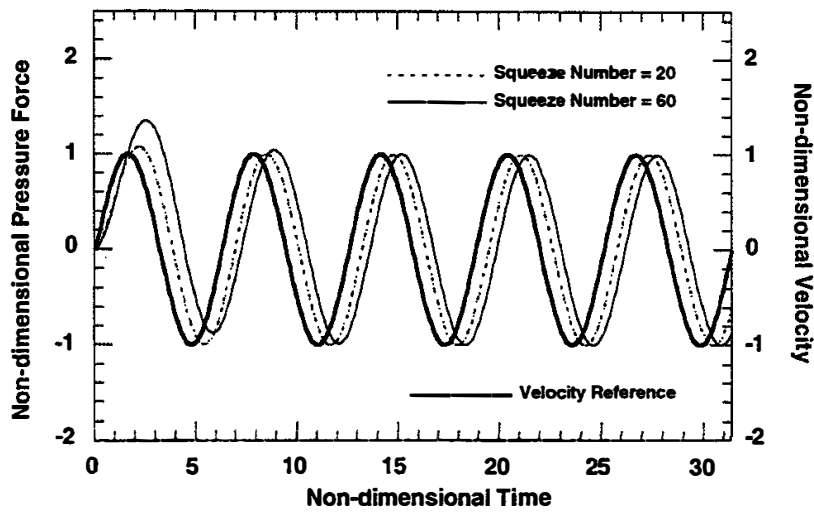

Fig. 3 Transient responses of the total back-force for two different squeeze numbers (20 and 60).

Fig. 4 is the schematic of a micro-accelerometer fabricated by the MIT MEMS group [10]. The proof mass is a $500 \mathrm{~mm}$ square plate supported $1 \mu \mathrm{m}$ above the substrate by flexible tethers. The simulated small-amplitude dynamical responses of the accelerometer are illustrated in Fig. 5. Curve 1 is a typical response without any air damping. Curve 2, which drops very fast, is the response with the air damping. In order to reduce damping, a perforated proof mass can be used. Fig. 6 is a contour plot of the transient pressure distribution for a perforated mass; Curve 3 of

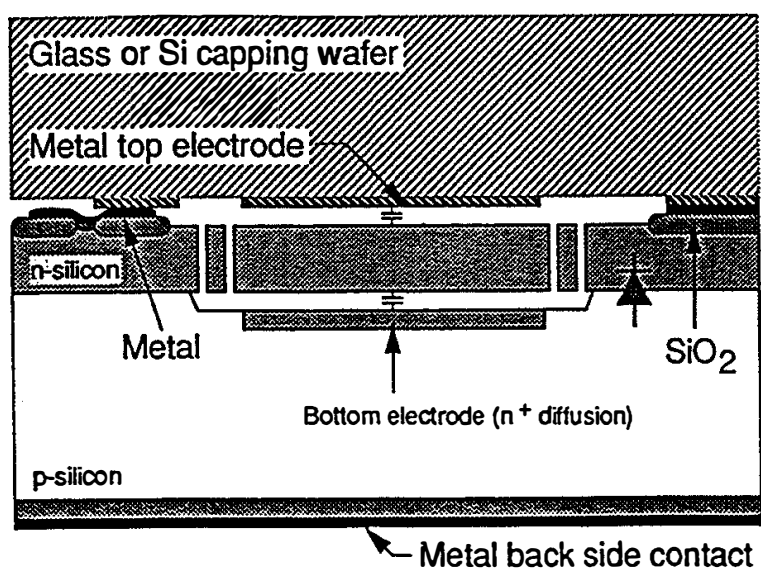

Fig. 4 Schematic view of a micro-accelerometer fabricated by the MIT MEMS group [10]. 
Fig. 5 is the corresponding response. Curves 2 and 3 show that the spring effect of the gas film results in a resonance shift. At low frequencies (low squeeze number), the system is overdamped because of highly viscous damping. At higher frequencies, the viscous damping decreases while the spring force increases (see Fig. 2). The resonance occurs at a high enough frequencies to make the resonan ce itself be underdamped.

Note that in many cases the gap thickness is too small to consider the air film as a continuum because of slip-flow condition at the boundaries. As a result, the damping effect is reduced. The viscosity can be modified to a smaller number to account for the slip-conditions at least to first order $[5,7,11]$.

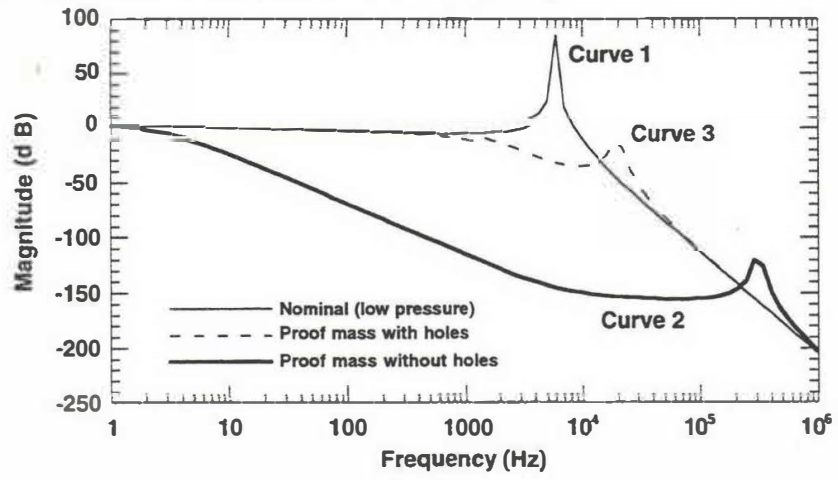

Fig. 5 Simulated dynamical responses of the microaccelerometer with and without air film damping effects.

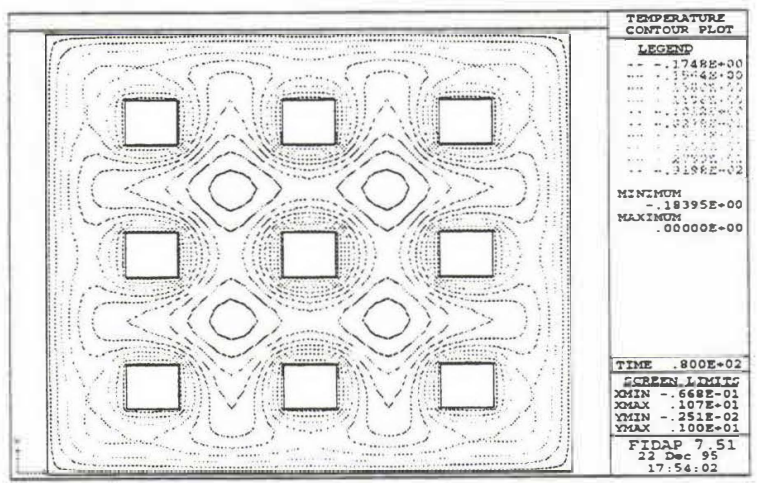

Fig. 6 Contour plot of the transient pressure distribution on a perforated proof mass of the accelerometer.

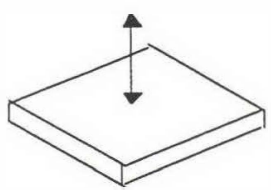

(A) Accelerometer

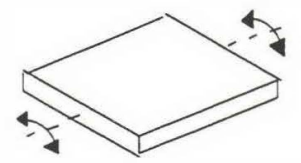

(C) Angular Rate Gyro

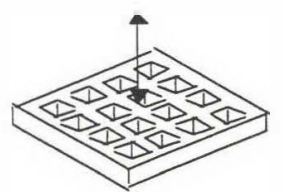

(A) Accelerometer with holes

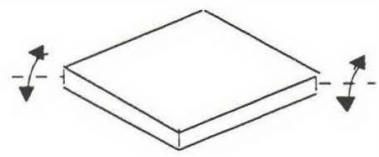

(D) Deformable Mirror Device
Fig. 7 Four examples of plate motion related to squeeze-film damping.

\section{LARGE AMPLITUDE MOTION}

In MEMS devices, the motion of the plate is not always a small amplitude oscillation. For example, the proof mass of the accelerometer shown in Fig. 4 will move a significant distance when undergoing a sudden change of acceleration. Therefore, the small amplitude oscillation model will not be valid in such a circumstance. Furthermore, some devices such as an angular rate gyro [12] use torsional motion, which is also significantly affected by squeezed-film damping, but which cannot be modeled using small amplitude motion. In order to deal with those problems, we have used the finite difference method to solve the isothermal Reynold's equation numerically. Fig. 7 shows four cases of squeeze-film damping problems with either large amplitude motions or non-parallel relative normal motions. The $3 \mathrm{D}$ surface and contour plots of pressure distributions for case (A) are shown in Fig. 8. These plots are the results at the time in the cycle at which the pressure reaches its maximum value. Fig. 9 is the dimensionless time response of the total back force for case (A), obtained by integrating the pressure on the plate over the plate area. The oscillation amplitude in this case is equal to $30 \%$ of the gap thickness. Because of the nonlinearity of Reynold's equation, the back force curve, which is normalized to the ambient pressure force (i.e., ambient pressure times the plate area), is not symmetric about the ambient pressure force ( $\mathrm{P}=1$ in the figure). When the plate moves down, the maximum back-force is about 1.2 times of the ambient pressure force; when plate moves up, the minimum back-force is about 0.9 of the ambient pressure force. Therefore
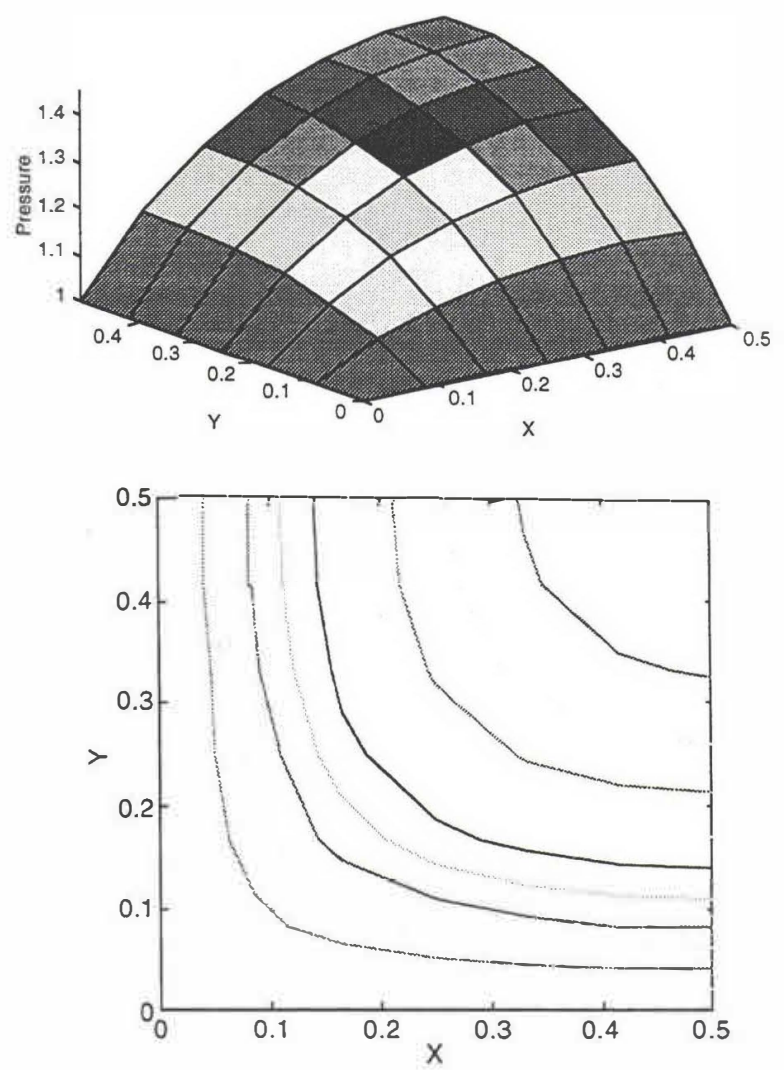

Fig. 8 3D-surface and contour plots of pressure distribution for one quarter of a square plate with large amplitude motion. 
the plate undergoes a larger pressure resistant force when the plate moves down. Note that the velocity curve in Fig. 9 has been normalized. The figure also indicates that the ransfer function between force and velocity is clearly nonlinear.

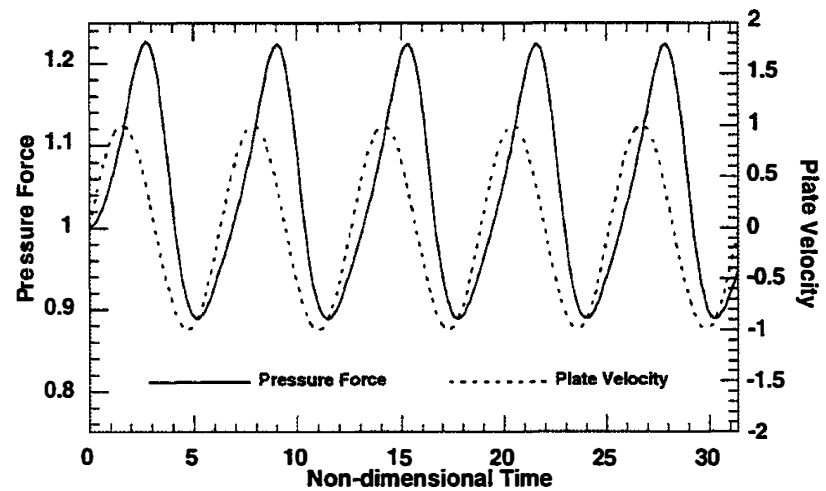

Fig. 9 Nonlinear time response of the total pressure back force for a square plate with a large amplitude oscillation.

Table 1 summarizes the maximum and minimum pressures on the plates for the four cases in Fig. 7 when the plates are sinusoidally oscillated in normal relative motion or torsional motion. Note that the pressure caused by torsional motion, which is also a potential second mode of motion for the accelerometer shown in Fig. 4, is much less than that caused by normal relative motion (case (A) in Fig. (7)) under ambient pressure. The design with the perforated plate also reduces the pressure significantly.

\begin{tabular}{|l|c|c|c|}
\hline \multicolumn{1}{|c|}{ CASE } & Max. d/ho & Max. P/Pa & Min. P/Pa \\
\hline (A) Accel. & 0.3 & 1.4484 & 0.7688 \\
\hline (B) Perforated Accel. & 0.3 & 1.1007 & 0.9079 \\
\hline (C) Gyro & 0.3 & 1.0902 & 0.9246 \\
\hline (D) DMD & 0.3 & 1.0635 & 0.9446 \\
\hline
\end{tabular}

Table 1 Summary of maximum and minimum pressure on the plates for the four cases in Fig. 7. Note that $\mathbf{d}$ is the plate oscillation amplitude, $\mathbf{h 0}$ is the initial gap thickness, $\mathbf{P}$ is the air film pressure on the plate, and $\mathbf{P a}$ is the ambient pressure. The oscillation amplitude for case (C) and (D) is the maximum displacement of the plate tip.

\section{SUMMARY AND CONCLUSION}

The numerical simulations of squeeze-film damping effects, based on the isothermal Reynold's equation, have been explored. The linearized model can be simulated by the finite element package FIDAP under small amplitude condition for plate geometries too complex for analytical solution. The viscous damping and spring effects of squeeze-films can be decoupled and modeled as frequency-dependent parameters for the small amplitude frequency response analysis of MEMS devices. An example, the dynamics of an accelerometer, is demonstrated. For the device operated in ambient pressure, the viscous damping effect dominates at low frequencies. The spring effect of the squeezed air film becomes significant at high frequency, and shifts the resonance by a large amount. The squeeze-film effect for large amplitude motions is simulated by finite difference codes. Four examples of different types of motions are demonstrated. The nonlinearity of the goveming equation gives rise to a nonlinear pressure back force on the plate. The maximum pressure back forces for each case under specific motion amplitude are summarized.

\section{ACKNOWLEDGMENTS}

This work was supported in part by Semiconductor Research Corporation and by ARPA. The authors wish to thank Prof. K. Breuer for helpful discussions, and Charles Hsu for providing the accelerometer example.

\section{REFERENCES}

[1] W. E. Langlois, "Isothermal Squeeze Films," Quarterly Applied Mathematics, Vol. XX, No. 2, 1962, pp. 131-150.

[2] J. J. Blech, "On Isothermal Squeeze Films," Journal of Lubrication Technology, Vol. 105, 1983, pp. 615-620.

[3] W. E. Newell, "The Miniaturization of Tuning Forks," Science, Vol. 161, 1968, pp. 1320-1326.

[4] H. Seidel, H. Riedel, R. Kolbeck, G. Miick, W. Kupke and M. Königer, "Capacitive Silicon Accelerometer with Highly Symmetrical Design," Sensors and Actuators, Vol. A21-23, 1990, pp. 312-315.

[5] M. Andrews, I. Harris and G. Turner, "A Comparison of Squeeze-film Theory with Measurements on A Microstructure," Sensors and Actuators A, Vol. A36, No. 2, March 1993, pp. 79-87.

[6] J. B. Starr, "Squeeze-film Damping in Solid-State Accelerometers," Tech. Digest, IEEE Solid State Sensor and Actuator Workshop, Hilton Head Island, SC, June 1990, pp. 44-47.

[7] T. Veijola, H. Kuisma, J. Lahdenperä and T. Ryhänen, "Equivalent-circuit model of the squeezed gas film in a silicon accelerometer," Sensors and Actuators A, Vol. A48, 1995, pp. 239-248.

[8] M. Novack, "Design and Fabrication of a Thin-film Micromachined Accelerometer," M.S. Thesis, Massachusetts Institute of Technology, 1992.

[9] FIDAP 7.0 manual, Fluid Dynamics International, Inc., 1993.

[10] C. Hsu and M. Schmidt, private communication.

[11] S. Dushman and J. M. Lafferty, Scientific Foundations of Vacuum Technique, Wiley, New York, 1962.

[12] J. Mizuno, K. Nottmeyer, C. Cabuz, T. Kobayashi, and M. Esashi, "Fabrication and Characterization of a Silicon Capacitive Structure for Simultaneous Detection of Acceleration and Angular Rate," Transducers '95 and Eurosensors IX, Stockholm, Sweden, June 1995, Vol. 2, pp. 679-682. 